\title{
Control of Plasma Confinement Layer for Effective Laser- peening
}

\author{
Yang Zhang $^{* 1}$, Noor Shahira binti Masroon ${ }^{1}$, Yoshinari Namba ${ }^{1}$, Miho Tsuyama ${ }^{1}$, Manabu Heya², and Hitoshi Nakano \\ ${ }^{1}$ Faculty of Science and Engineering, Kindai University, Japan \\ ${ }^{2}$ Faculty of Engineering, Osaka-sangyo University, Japan \\ *Corresponding author's e-mail: zhangyang@kindai.ac.jp
}

\begin{abstract}
Laser peening is a promising technique for cold working a metal sample by laser-induced shock waves. The amplitude of the shock waves can be increased by a plasma confinement layer, which suppresses the expansion of the laser-produced plasma. The properties of the plasma confinement layer affect the results of the laser peening treatment. In this study, an aqueous sucrose solution, which has a higher acoustic impedance than water, was selected as the plasma confinement layer. The concentration and temperature were varied to control the acoustic impedance and viscosity of the sucrose solution. It was found that when the concentration was around $20 \mathrm{wt} \%$ at a temperature of $60^{\circ} \mathrm{C}$, effective laser peening could be achieved.
\end{abstract}

DOI: 10.2961/jlmn.2021.02.2001

Keywords: sucrose, plasma confinement layer, laser peening, laser processing

\section{Introduction}

Laser peening is a technique for cold working a metal sample by shock waves generated due to laser-induced plasma pressure [1-3]. Since it has been shown in recent years that laser peening improves fatigue strength and prevents cracking due to stress corrosion, it has been put to practical use in various fields such as the aircraft, automobile, and nuclear industries. In addition, laser peening has the advantage of being a non-contact and localized surface treatment, and exhibits high process reproducibility.

Figure 1 shows the principle of laser peening [1]. When a metal sample placed in a liquid such as water is irradiated by a high-peak-power laser pulse, plasma is produced on the sample surface. The plasma is usually confined by a coating that is transparent to the laser, called the plasma confinement layer [4]. This confinement layer suppresses the rapid expansion of plasma away from the metal surface, so that a shock wave with a pressure of several gigapascals is generated. In addition, cavitation bubbles are produced by the absorption of laser energy in the plasma confinement layer, which then collapse and also generate a shock wave which propagates through the metal [5]. Solid substances begin to lose elasticity at pressures above the Hugoniot elastic limit and gradually become plastic. The shock wave pressure due to the plasma confinement layer and cavitation bubbles is high enough for the material to deform plastically [6]. Consequently, a compressive residual stress layer and a hardened layer are formed on the metal surface.

The effect of laser peening, $E_{\mathrm{LP}}$, which is usually expressed as the thickness of the plastically deformed layer, depends on the shock wave pressure and the shock loading time. That is, $E_{\mathrm{LP}} \propto \int_{0}^{\infty} P_{(t)} d t$, where $P_{(t)}$ is the shock wave pressure. The effect of laser peening depends on three factors: the material properties, the laser source, and the plasma confinement layer [7]. By optimizing these factors, the efficiency of laser peening can be improved. In this study, we attempt to optimize the plasma confinement layer for effective laser peening.
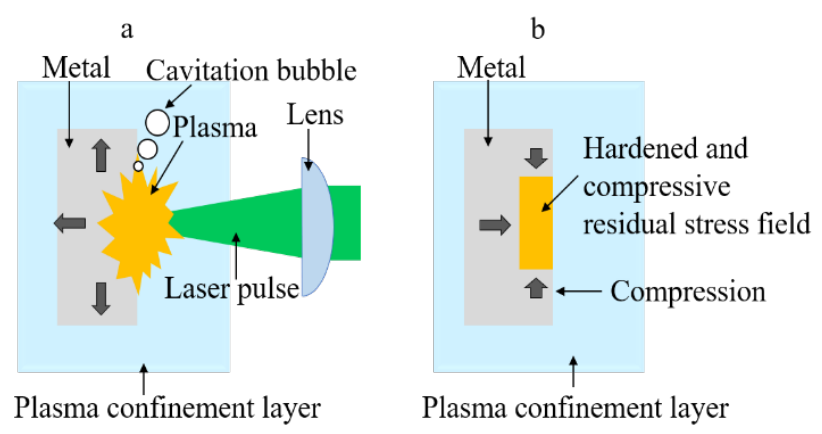

Fig. 1 Laser peening principle: (a) during laser irradiation, and (b) after laser irradiation.

\section{Plasma confinement layer}

The plasma confinement layer is a transparent medium capable of suppressing the expansion of laser-induced plasma and increasing the plasma pressure. Without this layer, the laser-induced plasma expands freely on the solid surface. However, when a plasma confinement layer is used, plasma expansion is suppressed due to the inertia of the layer, and the plasma is maintained in a high-pressure state on the sample surface. This high pressure of several gigapascals can modify the stress state in the near-surface region, can introduce beneficial compressive residual stress, and provides new opportunities for post-treatment processing [8]. Recently, there has been a considerable amount of research on different plasma confinement layers. The appropriate choice of confinement medium can significantly improve the efficiency of laser peening [9].

The ability of the plasma confinement layer to contain the plasma is strongly related to its effects on laser peening [4], and can be determined by the acoustic impedance $Z_{C}$, which is defined as the product of the density of the medium, $d_{\mathrm{L}}$, and the speed of sound, $v_{\mathrm{S}}$, in the medium [10]:

$$
Z_{\mathrm{C}}=d_{\mathrm{L}} \times v_{\mathrm{S}}
$$

Conditions needed for a good plasma confinement layer include high transmission of the laser light, no chemical 
reactivity toward the target material, high electrical resistivity, and high acoustic impedance. Based on these conditions, water has generally been used as the plasma confinement layer. A sucrose solution, which has a higher acoustic impedance and is more resistant to corrosion than water alone, has been used in our laboratory as a plasma confinement layer. As described by Tsuyama et al. in 2017 [11], the acoustic impedance of a sucrose solution increases with increasing concentration. The acoustic impedance of sucrose solutions with concentrations of $40 \mathrm{wt} \%$ and $60 \mathrm{wt} \%$ are 1.3 times and 1.5 times higher than that of water, respectively. As shown in Eq. (1), it is possible to control the acoustic impedance of the plasma confinement layer by changing the concentration of the sucrose solution. In our previous study [11], a sucrose solution was shown to be effective as a plasma confinement layer for laser peening.

However, it is important to note that a sucrose solution is viscous, and the viscosity of the plasma confinement layer affects the propagation of laser light. Figure 2 shows the viscosity of sucrose solutions at various temperatures as a function of concentration [12]. At the same temperature, the viscosity increases with concentration, and at the same concentration, the viscosity is reduced by increasing the temperature. Therefore, in this study, the effect of laser peening was investigated by controlling the temperature of the sucrose solution.

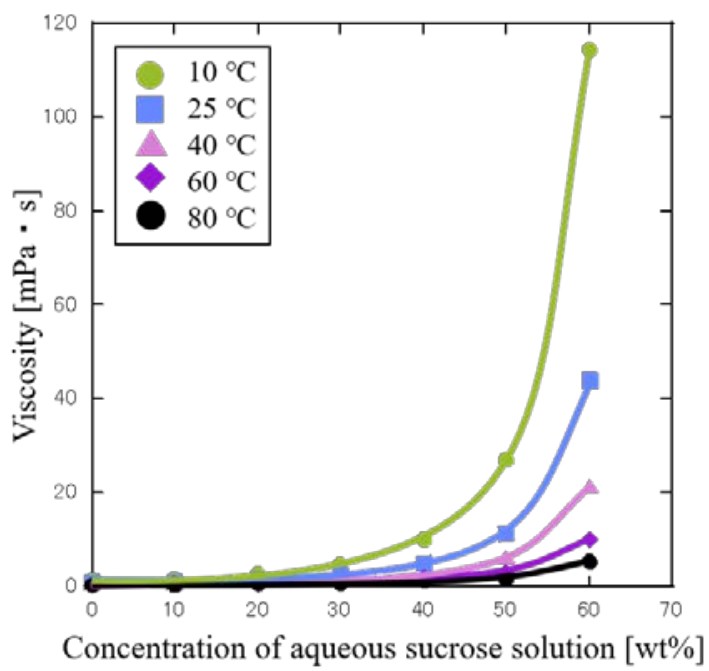

Fig. 2 Viscosity of aqueous sucrose solution as function of concentration and temperature.

\section{Experimental methods}

SUS316L, which is a form of steel that is widely used in industry, was used as the target material. The dimensions of the sample were $25 \mathrm{~mm} \times 25 \mathrm{~mm} \times 5 \mathrm{~mm}$. Commercially available metals are often processed by methods such as rolling, which cause strain accumulation. In order to standardize the initial properties of the target material and reduce the impact of pre-existing strain on the results, an annealing treatment was performed by heating the sample at $900^{\circ} \mathrm{C}$ for 3 hours in vacuum.

The optical setup for the laser peening experiments is shown in Fig. 3. As a laser light source, second harmonic radiation (wavelength $532 \mathrm{~nm}$ ) from a Nd:YAG laser was used. The pulse width and repetition rate were fixed at $4 \mathrm{~ns}$ and $10 \mathrm{~Hz}$, respectively. In this study, three lenses and a pinhole were used to deliver the energy to the target sample. The laser beam was passed through an energy attenuator and the pinhole, and was then focused on the sample in the direction normal to the surface by a lens with a focal length of $10 \mathrm{~cm}$. Adjusting the size of the pinhole allowed the diameter of the focal spot to be controlled. The laser intensity was set to $2 \mathrm{GW} / \mathrm{cm}^{2}$ using the attenuator. Part of the reflected light from the metal sample was transmitted through a mirror and imaged by a CCD camera to determine the dimensions of the focused spot. The coverage, referring to the number of laser shots per unit area, was set to $500 \%$ using an $\mathrm{XY}$ stage controlled by a PC.

For the plasma confinement layer, an aqueous sucrose solution was used. The concentration of the solution was varied in the range 5 to $60 \mathrm{wt} \%$ to control the acoustic impedance [11]. The temperature was also varied from 30 to $80^{\circ} \mathrm{C}$ to control the viscosity of the sucrose solution.

To evaluate the effect of laser peening, the surface workhardening and residual stress were determined using Vickers hardness and X-ray diffraction measurements, respectively. The residual stress in the depth direction was measured by etching the surface using electrolytic polishing.

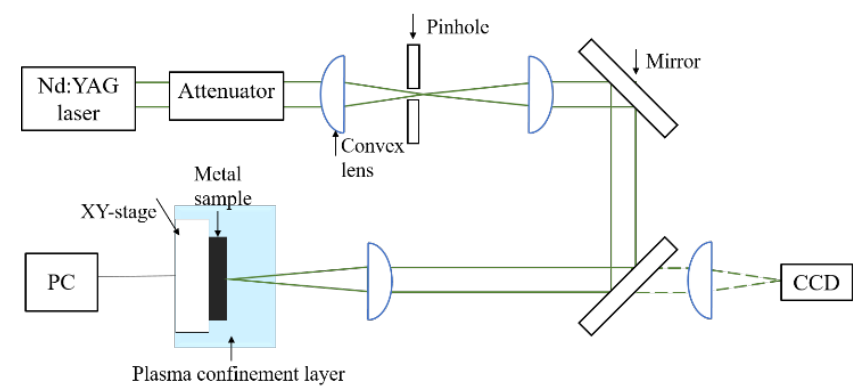

Fig. 3 Experimental setup for laser peening.

\section{Results}

Figure 4 shows the Vickers hardness difference of laserpeened samples as a function of the concentration of the sucrose solution. The hardness difference is defined as the hardness increase after laser peening. The temperature of the sucrose solution was fixed at $40^{\circ} \mathrm{C}$.

It can be seen the metal hardness initially increases with increasing concentration, reaches a maximum at a concentration of around $20 \mathrm{wt} \%$, and then decreases. To determine the influence of viscosity on laser peening, the temperature was changed to control the viscosity by referring to Fig. 2 .

Figure 5 shows the dependence of the Vickers hardness difference on the temperature of the sucrose solution. Six different concentrations of sucrose solution were used, namely, 5, 20, 30, 40, 50 and $60 \mathrm{wt} \%$. The temperature was changed in $10{ }^{\circ} \mathrm{C}$ increments from 30 to $80^{\circ} \mathrm{C}$.

From the results, it is found that the hardness of the sample surface depended on the temperature of the sucrose solution. At all concentrations, the hardness increased with increasing temperature and reached a maximum at $60^{\circ} \mathrm{C}$. Raising the temperature was shown to be effective in controlling the viscosity of the sucrose solution. 


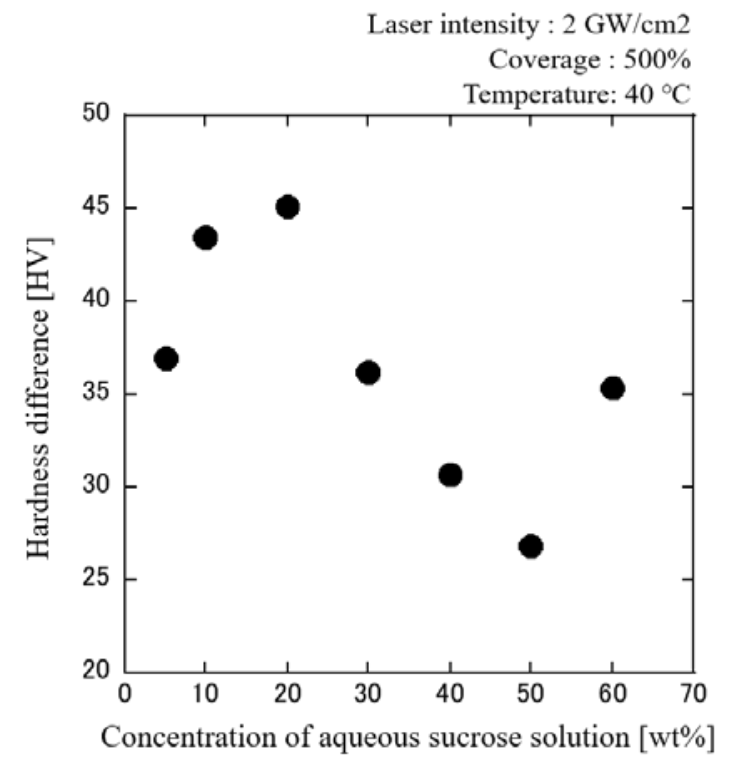

Fig. 4 Hardness difference as function of concentration of aqueous sucrose solution.

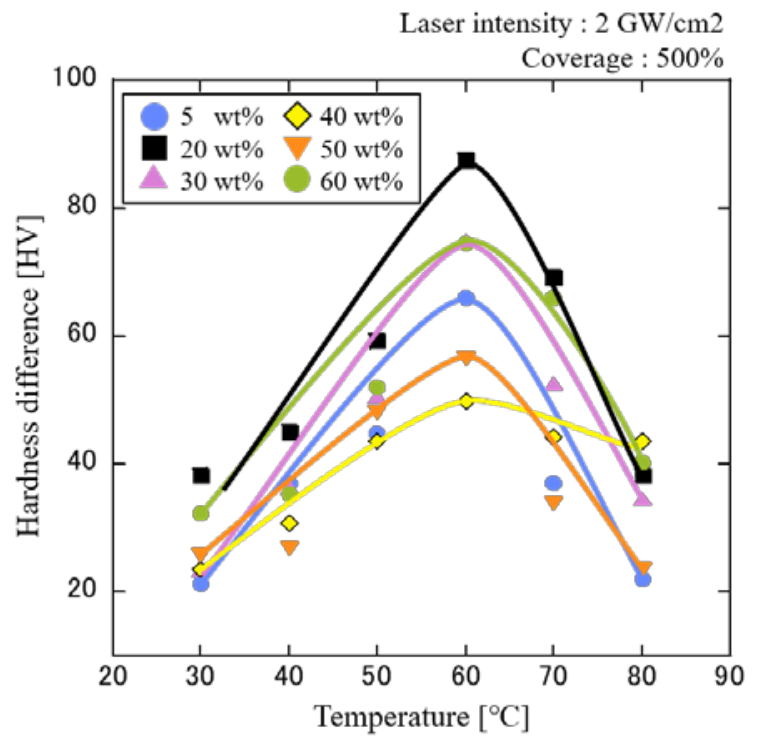

Fig. 5 Hardness difference as function of temperature of aqueous sucrose solution.

To estimate the induced residual stress, the samples were characterized by X-ray diffraction. Figure 6 shows the residual stress profiles in the sample depth direction. Since the penetration depth of X-rays in the sample is at most several tens of microns, so that only the stress in the near-surface region can be measured, the residual stress distribution was accurately measured by repeatedly electropolishing the sample with a step size of $20 \mu \mathrm{m}$. Positive and negative values on the vertical axis indicate tensile and compressive stress, respectively. The experiment was conducted at room temperature (about $25^{\circ} \mathrm{C}$ ) and also at $60^{\circ} \mathrm{C}$, where the highest Vickers hardness is seen in Fig. 5.

In Fig. 6, it can be seen that although the residual stress was tensile on the surface of the sample (depth $0 \mu \mathrm{m}$ ), compressive stress was produced inside the sample. The compressive residual stress reached a peak at around a depth of $20 \mu \mathrm{m}$. At room temperature, the maximum value was about
$-200 \mathrm{MPa}$, while at $60^{\circ} \mathrm{C}$ it increased to $-350 \mathrm{MPa}$. The compressive residual stress at $60^{\circ} \mathrm{C}$ was greater than that at room temperature, which also indicates that raising the temperature to control the viscosity is effective for laser peening.

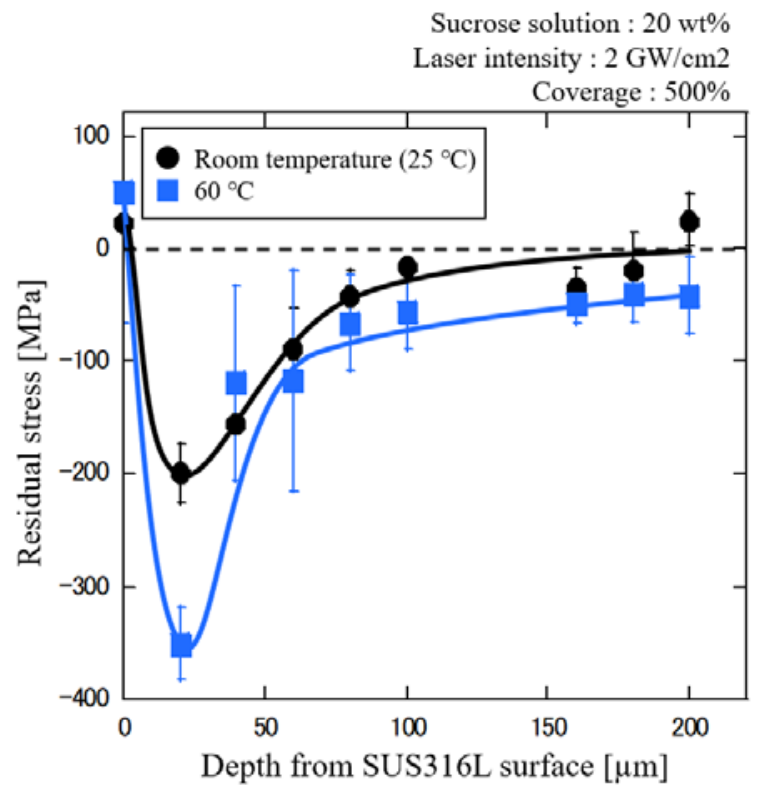

Fig. 6 Residual stress as function of depth from sample surface.

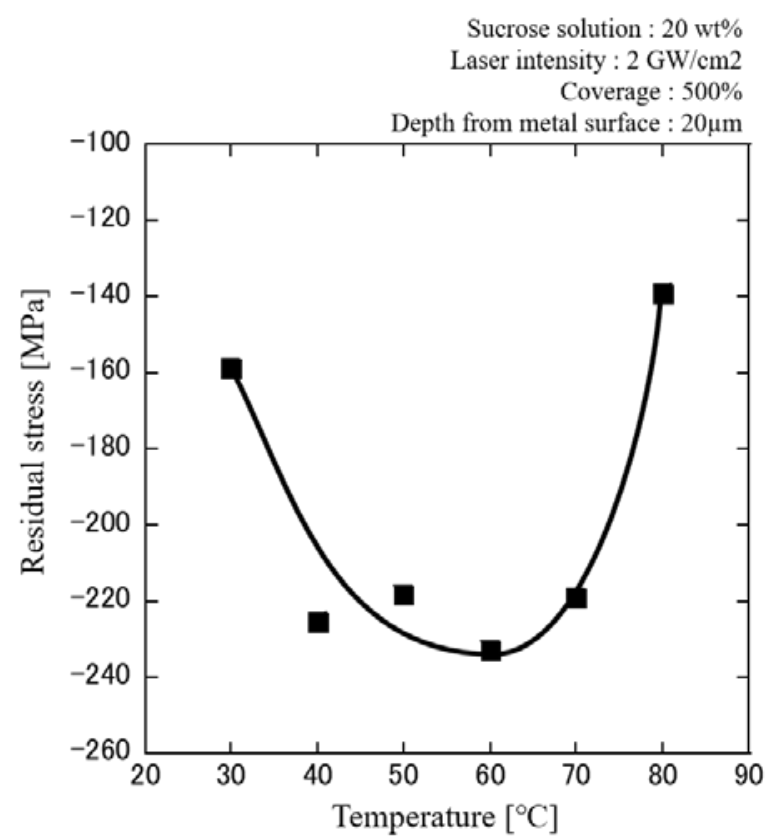

Fig. 7 Residual stress as function of temperature of aqueous sucrose solution.

Figure 7 shows the residual stress as a function of the temperature of the sucrose solution. The concentration of the solution was $20 \mathrm{wt} \%$, which exhibits the highest hardness difference in Fig. 4. The data were taken at a depth of $20 \mu \mathrm{m}$ from the sample surface, where the highest residual stress is seen in Fig. 6.

It can be seen that the magnitude of the compressive residual stress depends on the temperature of the sucrose solution, exhibiting a maximum at around $60^{\circ} \mathrm{C}$, which is similar to the results for the Vickers hardness shown in Fig. 5. 


\section{Discussion}

Using an aqueous sucrose solution as a plasma confinement layer during laser peening, the metal hardness exhibited a maximum for a sucrose solution concentration of around $20 \mathrm{wt} \%$, and then decreased at higher concentrations. The initial increase in metal hardness with increasing sucrose solution concentration is thought to be due to an increase in the acoustic impedance. However, this cannot explain the decrease in hardness at concentrations over $20 \mathrm{wt} \%$. The reason for this decrease may be related to the viscosity of the solution. When the concentration is higher than 20 wt $\%$, the viscosity of the solution is high so that its movement is suppressed, and the rate of bubble growth and collapse is significantly reduced. The cavitation bubbles rise slowly toward the surface of the solution and stay in the laser beam path, which causes laser pulse scattering and eventually reduces the effect of laser peening [10].

Although the effect of viscosity can be reduced by increasing the temperature, the density of the solution will decrease, resulting in lower acoustic impedance and ultimately a reduction in the effect of laser peening.

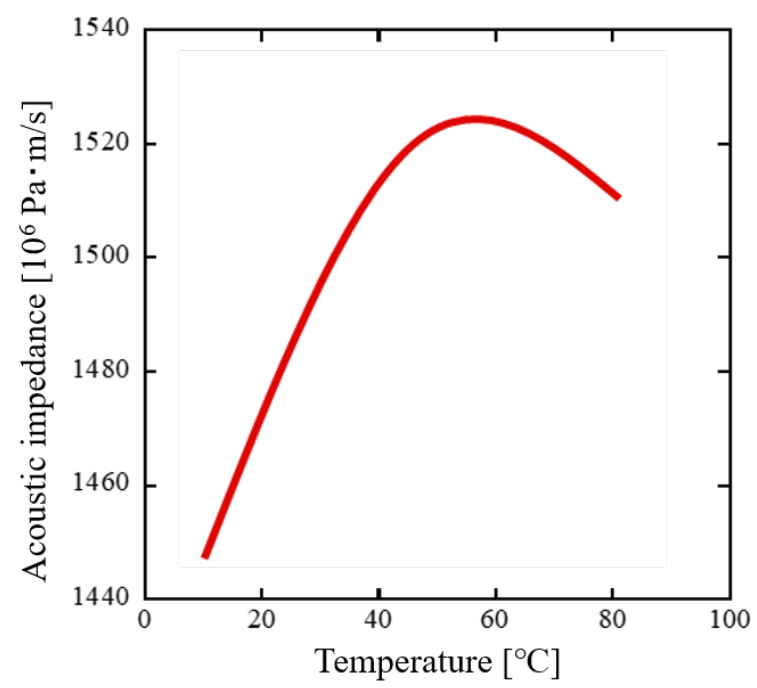

Fig. 8 Acoustic impedance of water as function of temperature.

As shown in Figs. 5 and 7, the Vickers hardness and compressive residual stress exhibited a maximum at $60^{\circ} \mathrm{C}$, and this is thought to be related to the acoustic impedance of water. Figure 8 shows the dependence of the acoustic impedance of water on the temperature [13]. It can be seen that the acoustic impedance increases with increasing temperature, and reaches a maximum at around $60^{\circ} \mathrm{C}$, similar to the trends for hardness and residual stress.
Consequently, it is important to balance the effects of viscosity and acoustic impedance to find the most suitable conditions for effective laser peening.

\section{Conclusion}

In this study, the effect on laser peening of controlling the plasma confinement layer (aqueous sucrose solution) by changing the concentration and temperature was investigated.

It was found that the resulting metal hardness exhibits a maximum for a sucrose solution concentration of $20 \mathrm{wt} \%$, but decreases at high concentrations due to increased viscosity. Both the hardness and the residual stress increase with temperature up to $60^{\circ} \mathrm{C}$, similar to the acoustic impedance of water. In conclusion, controlling the concentration and temperature of the sucrose solution used as a plasma confinement layer is an effective means of achieving efficient laser peening.

\section{References}

[1] R. Fabbro, P. Peyre, L.Berthe, and X. Scherpereel: J. Laser Appl., 10, (1998) 265.

[2] K. Ding and L. Ye: "Laser Shock Peening” ed. by CRC Press (Boca Raton, 2006).

[3] Y. Sano, K. Akita, K. Masaki, Y. Ochi, I. Altenberger, and B. Scholtes: J. Laser Micro/Nanoeng., 1, (2006) 161.

[4] X. Hong, S. Wang, D. Guo, H. Wu, J. Wang, Y. Dai, X. Xia, and Y. Xie: Opt. Lasers Eng., 29, (1998) 447.

[5] H. Soyama: J. Mater. Process. Tech., 269, (2019) 65.

[6] S. Kalainathan, and S. Prabhakaran: Opt. Laser Technol., 81, (2016) 137.

[7] M. Tsuyama, Y. Kodama, Y. Miyamoto, I. Kitawaki, M. Tsukamoto, and H. Nakano: J. Laser Micro/Nanoeng., 11, (2016) 227.

[8] N. Kalentics, A. Burn, M. Cloots, and R. E. Logé: Int. J. Adv. Manuf. Technol., 101, (2019) 1247.

[9] K. Elango, J. S. Hoppius, L. M. Kukreja, A. Ostendorf, and E. J. Gurevich: Surf. Coat. Technol., 397, (2020) 125988

[10] M. Tsuyama, N. Ehara, K. Yamashita, M. Heya, and H. Nakano: Appl. Phys. A Mater. Sci. Process., 124, (2018) 249.

[11] M. Tsuyama, N. Ehara, K. Yamashita, M. Heya, and H. Nakano: Rev. Laser Eng., 45, (2017) 658. (in Japanese)

[12] V.R.N. Telis, J. Telis-Romero, H.B. Mazzotti, and A.L. Gabas: Int. J. Food Prop., 10, (2007) 185.

[13] M. Chavez, V. Sosa, and R. Tsumura: J. Acoust. Soc. Am., 77, (1985) 420.

(Received: June 10, 2021, Accepted: August 22, 2021) 